\title{
TIME REVERSAL MIMO RADAR: IMPROVED CRB AND ANGULAR RESOLUTION LIMIT
}

\author{
Foroohar Foroozan* ${ }^{*}$ Amir Asif*, and Rémy Boyer ${ }^{\dagger}$ \\ ${ }^{*}$ Computer Science and Engineering, York University, Toronto, ON, Canada M3J 1P3 \\ ${ }^{\dagger}$ Laboratoire des Signaux et Système, Université Paris-Sud XI, France
}

\begin{abstract}
The paper derives closed form (nonmatrix) expression for the deterministic Cramér-Rao bound (CRB) for the direction-of-arrival associated with a target embedded in a noisy, multipath channel using the time reversal (TR) MIMO system. By incorporating the TR built-in adaptive waveform processing feature to reshape the MIMO probing signals, we prove that the CRBs for the direction of arrival can be improved in ways not foreseen with the conventional MIMO radars. A second contribution of the paper is the analytical derivation of the Angular Resolution Limit (ARL) defined as the minimal separation between two targets to be separately resolved by the MIMO radar. At high signal-to-noise ratio, we show that the TR ARL inherits the properties of the TR CRB and is superior to its conventional counterpart by a factor proportional to the order of the channel multipath.
\end{abstract}

Index Terms - Time Reversal, MIMO radar, CRB and ARL.

\section{INTRODUCTION}

Time reversal (TR) utilizes the reciprocity property of wave propagation in a time-invariant medium. TR based methods time reverse, energy normalize, and retransmit (mathematically or physically) a previously recorded backscatter of a probing signal reflected from the dispersive medium. As the process is repeated, the TR signal becomes highly focused on the sources (targets) producing backscatters. This phenomenon is referred to as super-resolution focusing [1-3], which among other factors depends on the physical aperture of the transmitter-receiver array and the medium. A complex medium creates the so-called multipath effect and significantly increases the effective aperture of the transmitter-receiver array. Indeed, TR harnesses multipathing to enhance focusing resolution beyond the classical diffraction limit. This paper applies TR to multiple-input/multiple-output (MIMO) radars for improving the accuracy of the estimation algorithms associated with an aerospace target. Indeed, several researchers have reported that taking advantage of the super-resolution focusing property of TR improves the detection accuracy $[4,5]$ in radars. Previously, we have applied TR for radar target localization using both single-input/multiple-output (SIMO) [6,7] and MIMO radar systems [8-10].

MIMO radar has the ability to transmit multiple and potentially different probing signals [11]. The resulting waveform diversity in MIMO radars enables superior performance including improved target identifiability [12], clutter/interference rejection that includes fading mitigation [13], and interference suppression [14]. The advantages of a MIMO radar system with both colocated and widely separated antenna elements are further investigated in [15] to offer improved resolution and higher sensitivity towards detecting moving targets. In this work, we focus on the case of colocated antennas in a MIMO setup, where the transmit waveforms are adjusted according to the channel characteristics using TR. The first section of the paper derives and analyzes closed form (nonmatrix) expressions of the deterministic Cramer-Rao bounds (CRB) for the direction of arrival associated with a target embedded in a medium that is contaminated by multipath clutter and background noise using the TR/MIMO framework. To assess the accuracy of the estimation of the target parameters, the CRB [16] is a universally accepted tool and provides the optimal accuracy achievable by any unbiased estimator of the target parameters. What distinguishes the CRB derived in this paper from the great majority of existing derivations in literature, e.g., [17-22], is that the TR based MIMO CRBs are formulated in a multipath environment. By incorporating the TR built-in adaptive waveform processing feature to design the TR probing signals, our intuition is that the CRB can be further improved in ways which were not foreseen with the non-adaptive waveforms (referred to as conventional) MIMO radars. The second section of the paper focuses on the analytical derivation and analysis of the Angular Resolution Limit (ARL) [21], which is the minimal separation between two closely located targets at which they can be resolved separately. Deriving the ARL of a radar system is a fundamental problem [23] and has previously been formulated in a clutter free environment [21]. This paper considers multipath channels. For the simplified case of two targets and a single interference source, we show that the TR ARL is lower than the conventional ARL by a factor that depends on the strength of the multipath at equal Signal-to-Interference-Noise ratio (SINR). To the best of our knowledge and among all existing literature on the $\mathrm{CRB}$ and ARL surveyed by us, no such contribution has been made in the context of the ARL for the TR MIMO radars.

The paper is organized as follows. Section 2 defines the notation and derives the mathematical formulation for the conventional MIMO system followed by the TR/MIMO system using a noisy 3way parallel factor analysis (PARAFAC) model [19,24]. Section 3 derives the CRBs for the TR MIMO radar, while Section 4 formulates the TR ARL. Results from numerical examples are discussed in Section 5. Finally, Section 6 concludes the paper.

\section{PROBLEM SETUP}

Standard MIMO: We consider a colocated MIMO radar system with an equal number $P$ of transmit and receive antenna elements monitoring $L$ moving targets modeled as narrowband point-sources in the far field [11]. The received signal [25] resulting from the $k$ 'th, $(0 \leq k \leq(K-1))$ transmitted pulse, with $K$ being the number of pulses in one coherent pulse interval (CPI), is

$$
\mathbf{R}_{k}=\sum_{l=1}^{L} \alpha_{l} e^{j 2 \pi f_{l} k} \mathbf{a}_{R}\left(\zeta_{l}\right) \mathbf{a}_{T}^{T}\left(\zeta_{l}\right) \mathbf{F}+\mathbf{N}_{k},
$$

where $\alpha_{l}$ and $f_{l}$ denote, respectively, the reflection coefficient and the normalized Doppler frequency associated with the l'th target. The target velocity from path $l$ is denoted by $v_{l}$ and the radar pulse 
period by $T_{p}$ and the wavelength of the propagating wave by $\lambda$, then $f_{l}=2 T_{p} v_{l} / \lambda$. Let $Q$ be the number of samples in each CPI, then the known $P \times Q$ probing signal matrix $\mathbf{F}$ is defined by $\mathbf{F}=\left[\mathbf{f}_{1} \cdots \mathbf{f}_{P}\right]^{T}$ where $\mathbf{f}_{i}=\left[F_{i}(0) \cdots F_{i}(Q-1)\right]^{T}$, whereas the $P \times Q$ noise matrix for the $k$ 'th pulse is denoted by $\mathbf{N}_{k}$. The transmit and receive steering vectors are denoted by $\mathbf{a}_{T}($.$) and \mathbf{a}_{R}($.$) with the i$ 'th elements of these vectors given by $\left[\mathbf{a}_{T}\left(\zeta_{l}\right)\right]_{i}=\exp \left(j(2 \pi / \lambda) \zeta_{l} d_{i}^{T}\right)$ and $\left[\mathbf{a}_{R}\left(\zeta_{l}\right)\right]_{i}=\exp \left(j(2 \pi / \lambda) \zeta_{l} d_{i}^{R}\right)$, respectively, where $\zeta_{l}=\sin \left(\theta_{l}\right)$ considering that the target direction of arrival (DOA) is denoted by $\theta_{l}$. Notations $d_{i}^{T}$ and $d_{i}^{R}$ are the distances between the reference sensor and the $i$ 'th transmission and reception arrays, respectively. We assume that the transmitted signals are orthogonal, then relations $\mathbf{F F}^{H}=\mathbf{F}^{*} \mathbf{F}^{T}=Q \mathbf{I}_{P}$ hold true. After matched filtering, Eq. (1) is given as

$$
\mathbf{Y}_{k}=\frac{1}{\sqrt{Q}} \mathbf{R}_{k} \mathbf{F}^{H}=\sum_{l=1}^{L} \overbrace{\sqrt{Q} \alpha_{l}}^{\tilde{\alpha}_{l}} e^{j 2 \pi f_{l} k} \mathbf{a}_{R}\left(\zeta_{l}\right) \mathbf{a}_{T}^{T}\left(\zeta_{l}\right)+\mathbf{V}_{k},
$$

where $\mathbf{V}_{k}=1 / \sqrt{Q} \mathbf{N}_{k} \mathbf{F}^{H}$ denotes the noise matrix after matched filtering. Following the CanDecomp/Parafac model [24]

$$
\mathbf{y}=\left[\operatorname{vec}\left(\mathbf{Y}_{0}\right)^{T} \cdots \operatorname{vec}\left(\mathbf{Y}_{K-1}\right)^{T}\right]^{T}=\sum_{l=1}^{L} \tilde{\alpha}_{l} \mathbf{g}_{l}+\mathbf{v},
$$

where $\mathbf{g}_{l}=\mathbf{a}\left(f_{l}\right) \otimes \mathbf{a}_{T}\left(\zeta_{l}\right) \otimes \mathbf{a}_{R}\left(\zeta_{l}\right)$, the Doppler frequency vector $\mathbf{a}\left(f_{l}\right)=\left[\begin{array}{ll}1 & e^{2 j \pi f_{l}} \cdots e^{2 j \pi f_{l}(K-1)}\end{array}\right]^{T}$, and $\otimes$ denotes the Kronecker product. Notation $\mathbf{v}=\left[\operatorname{vec}\left(\mathbf{V}_{0}\right)^{T} \cdots \operatorname{vec}\left(\mathbf{V}_{K-1}\right)^{T}\right]^{T}$ vectorizes the noise matrices of $K$ pulses. Let the observation noise before matched filtering be complex circular Gaussian independent and identically distributed samples with zero mean and covariance of $\sigma_{v}^{2} \mathbf{I}$. Due to the orthogonality of the probing signals, one can show that $\mathcal{E}\left(\mathbf{v} \mathbf{v}^{H}\right)=\sigma_{v}^{2} \mathbf{I}_{K P^{2}}$ [11] with $\mathcal{E}\{$.$\} being the expected$ operator. Without loss of generality, we assume that the targets of interest are the first and the second indices of $l$ (i.e., with $l=1,2$ ) and the rest of $L-2$ signals are clutter. Then, Eq. (3) is given as

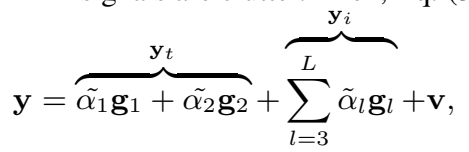

where the signal of interest $\mathbf{y}_{t}$ from the two targets is deterministic. In general, the clutter amplitudes $\tilde{\alpha}_{l}$ are assumed to be zero-mean complex circular Gaussian independent random variables. Due to the orthogonality of the transmitted waveforms and the central limit theorem, it can be shown that observations $\mathbf{y}$ have circular complex Gaussian distribution with mean $\mathbf{y}_{t}$ and covariance $\overline{\mathbf{\Sigma}}$.

TR MIMO: In TR, the backscatter of the signals are reversed in time, energy normalized, conjugated, and re-transmitted a second time into the medium. The transmitted signal $\mathbf{R}_{k}^{\mathrm{TR}}=c_{k}\left\{\mathbf{R}_{k}^{*}\right\}_{P \times \text { end:-1:1 }}$ where $c_{k}$ is the energy normalization factor for the $k$ 'th pulse. Here, the Matlab notation $\{$ end:- $1: 1\}$ is used for showing the time reversal operation. Similar to (1), the $k$ 'th received signal in the TR stage is

$$
\begin{gathered}
\mathbf{X}_{k}=\sum_{l^{\prime}=1}^{L} \alpha_{l} e^{j 2 \pi f_{l^{\prime}} k} \mathbf{a}_{T}\left(\zeta_{l^{\prime}}\right) \mathbf{a}_{R}^{T}\left(\zeta_{l^{\prime}}\right) \mathbf{R}_{k}^{\mathrm{TR}}+\mathbf{M}_{k}=c_{k} \sum_{l^{\prime}=1}^{L} \sum_{l=1}^{L} \\
\alpha_{l}^{*} \alpha_{l^{\prime}} e^{j 2 \pi\left(f_{l}+f_{l^{\prime}}\right) k} \mathbf{a}_{T}\left(\zeta_{l^{\prime}}\right) \mathbf{a}_{R}^{T}\left(\zeta_{l^{\prime}}\right) \mathbf{a}_{R}^{*}\left(\zeta_{l}\right) \mathbf{a}_{T}^{H}\left(\zeta_{l}\right) \mathbf{F}^{\mathrm{TR}}+\mathbf{W}_{k}(5)
\end{gathered}
$$

where $\mathbf{F}^{\mathrm{TR}}$ is the same as $\mathbf{F}^{*}$ except for time reversal. The accumulated observation noise $\mathbf{W}_{k}$ takes both $\mathbf{M}_{k}$ and $\mathbf{N}_{k}$ into account. Due to the TR focusing property, Eq. (5) is approximated as $[1,26]$.

$$
\mathbf{X}_{k} \approx c_{k} P \sum_{l=1}^{L}\left|\alpha_{l}\right|^{2} e^{j 2 \pi 2 f_{l} k} \mathbf{a}_{T}\left(\zeta_{l}\right) \mathbf{a}_{T}^{H}\left(\zeta_{l}\right) \mathbf{F}^{\mathrm{TR}}+\mathbf{W}_{k} .
$$

Following the procedure used in conventional matched filtering, we apply the matched filter to the TR observation given in Eq. (6) by multiplying with $\mathbf{F}^{\mathrm{TR} H}$. Using the orthogonality of $\mathbf{F}$, it can be shown that $\mathbf{F}^{\mathrm{TR}} \mathbf{F}^{\mathrm{TR} H}=Q \mathbf{I}_{p}$. Using this equality and after matched filtering of the TR observations (Eq. (6)), we have

$\mathbf{Z}_{k}=\frac{1}{\sqrt{Q}} \mathbf{X}_{k} \mathbf{F}^{\mathrm{TR} H}=\sum_{l=1}^{L} \overbrace{P \sqrt{Q}\left|\alpha_{l}\right|^{2}}^{{\tilde{\alpha_{l}}}^{\mathrm{TR}}} c_{k} e^{j 2 \pi 2 f_{l} k} \mathbf{a}_{T}\left(\zeta_{l}\right) \mathbf{a}_{T}^{H}\left(\zeta_{l}\right)+\mathbf{U}_{k}$.

Stacking the TR returns of all $k, 0 \leq k \leq(K-1)$ pulses in vector $\mathbf{z}=\left[\operatorname{vec}\left(\mathbf{Z}_{0}\right)^{T} \cdots \operatorname{vec}\left(\mathbf{Z}_{K-1}\right)^{T}\right]^{T}$, the TR model is represented as

$$
\mathbf{z}=\sum_{l=1}^{L}{\tilde{\alpha_{l}}}^{\mathrm{TR}} \mathbf{g}_{l}^{\mathrm{TR}}+\mathbf{u}
$$

in which $\mathbf{g}_{l}^{\mathrm{TR}}=\mathbf{a}^{\mathrm{TR}}\left(f_{l}\right) \otimes \mathbf{a}_{T}\left(\zeta_{l}\right) \otimes \mathbf{a}_{T}^{*}\left(\zeta_{l}\right)$ and $\mathbf{a}^{\mathrm{TR}}\left(f_{l}\right)=$ $\left[\begin{array}{lll}c_{0} & c_{1} e^{2 j \pi 2 f_{l}} \cdots c_{K-1} e^{2 j \pi 2 f_{l}(K-1)}\end{array}\right]^{T}$. Notation $\mathbf{u}$ vectorizes all the noise matrices of $K$ pulses. Following the discussion for the standard MIMO observations, one can show that TR observations $\mathbf{z}$ is Gaussian with a covariance of $\overline{\boldsymbol{\Sigma}}^{\mathrm{TR}}=\sigma_{u}^{2} \boldsymbol{\Pi}^{\mathrm{TR}}$, where $\boldsymbol{\Pi}^{\mathrm{TR}}$ is a positive definite matrix and $\sigma_{u}^{2}$ is the noise variance.

\section{DETERMINISTIC CRB FOR THE TR MIMO RADAR}

In this section, we derive the TR CRB expressions assuming that one target to be present. Rest of the sources are considered as interference. Based on Eq. (7), the TR observations become

$$
\mathbf{z}=\overbrace{{\tilde{\alpha_{1}}}^{\mathrm{TR}} \mathbf{g}_{1}^{\mathrm{TR}}}^{\mathbf{z}_{t}}+\sum_{l=2}^{L}{\tilde{\alpha_{l}}}^{\mathrm{TR}} \mathbf{g}_{l}^{\mathrm{TR}}+\mathbf{u} .
$$

The vector of unknown parameters is $\boldsymbol{\Phi}=\left[\begin{array}{l}\boldsymbol{\Phi}_{t}^{T} \boldsymbol{\Phi}_{i}^{T}\end{array}\right]^{T}$, where $\boldsymbol{\Phi}_{t}=$ $\left[\theta_{1}, v_{1}, \tilde{\alpha}_{1}^{\mathrm{TR}}\right]^{T}$ are the deterministic parameters of interest. The nuisance parameters are $\boldsymbol{\Phi}_{i}=\left[\theta_{2}, \cdots, \theta_{L}, \tilde{\alpha}_{2}^{\mathrm{TR}}, \cdots, \tilde{\alpha}_{L}^{\mathrm{TR}}\right]^{T}$, where the interferences are assumed stationary. We assume that the normalization factor is the same for all $K$ pulses (i.e. $c_{k}=c$ for $\forall k$ ), which is taken out from $\mathbf{a}^{\mathrm{TR}}\left(v_{l}\right)$ and considered in the TR attenuation factors.

\subsection{Deterministic TR CRB for Gaussian Interference}

The deterministic CRB for a Gaussian interference [27] is

$$
\mathbf{J}^{\mathrm{TR}^{-1}}=\frac{2}{\sigma_{u}^{2}} \mathcal{R}\left\{\left(\frac{\partial \mathbf{z}_{t}}{\partial \boldsymbol{\Phi}_{t}}\right)^{H} \overline{\mathbf{\Sigma}}^{\mathrm{TR}^{-1}}\left(\frac{\partial \mathbf{z}_{t}}{\partial \boldsymbol{\Phi}_{t}}\right)\right\},
$$

where $\overline{\boldsymbol{\Sigma}}^{\mathrm{TR}}=\sigma_{u}^{2} \boldsymbol{\Pi}^{\mathrm{TR}}$ is the covariance of the clutter plus noise in the TR phase. Using the three auxiliary vectors

$\mathbf{e}_{1} \triangleq \boldsymbol{\Pi}^{\mathrm{TR}-1 / 2} \mathbf{g}_{1}^{\mathrm{TR}}, \quad \mathbf{e}_{\theta_{1}} \triangleq \boldsymbol{\Pi}^{\mathrm{TR}-1 / 2} \mathbf{g}_{\theta_{1}}^{\mathrm{TR}}, \quad \mathbf{e}_{v_{1}} \triangleq \boldsymbol{\Pi}^{\mathrm{TR}-1 / 2} \mathbf{g}_{v_{1}}^{\mathrm{TR}}$,

where $\boldsymbol{\Pi}^{\mathrm{TR}} \mathrm{R}^{-1 / 2}$ is the inverse of the square root of $\boldsymbol{\Pi}^{\mathrm{TR}}$ and $\mathbf{g}_{\theta_{1}}^{\mathrm{TR}}$ and $\mathbf{g}_{v_{1}}^{\mathrm{TR}}$ are the partial derivative of $\mathbf{g}_{1}^{\mathrm{TR}}$ with respect to $\theta_{1}$ and $v_{1}$, respectively. The closed form expression of the TR CRB for the direction parameter is given as (Eq. (11) of [19])

$$
\begin{aligned}
\operatorname{CRB}^{\mathrm{TR}}\left(\theta_{1}\right) & =\frac{1}{2 \mathcal{C}_{\mathrm{TR}}^{1}} \frac{\left\|\mathbf{e}_{v_{1}}\right\|^{2}}{\rho} \sin ^{2}(\Theta), \text { with } \\
\rho & =\left\|\mathbf{e}_{\theta_{1}}\right\|^{2}\left\|\mathbf{e}_{v_{1}}\right\|^{2} \sin ^{2}(\Theta) \sin ^{2}(\Omega)-\left(\frac{\Re\left\{\mathbf{e}_{1}^{H} \mathbf{A} \mathbf{e}_{1}\right\}}{\left\|\mathbf{e}_{1}\right\|^{2}}\right)^{2}
\end{aligned}
$$


where $\mathcal{C}_{\mathrm{TR}}^{1}=\frac{c^{2}\left|\tilde{\alpha 1}_{1}^{\mathrm{TR}}\right|^{2}}{\sigma_{u}^{2}}$ and $\mathbf{A}=\left(\mathbf{e}_{\theta_{1}}^{H} \mathbf{e}_{v_{1}}\right) \mathbf{I}-\mathbf{e}_{\theta_{1}} \mathbf{e}_{v_{1}}^{H}$. Also, in Eq. (10), we have introduced two distances as in [19]: $\operatorname{dist}\left(\mathcal{E}_{\theta_{1}}, \mathcal{E}\right)=$ $\sin ^{2}(\Omega)=1-\left(\left|\mathbf{e}_{\theta_{1}}^{H} \mathbf{e}_{1}\right|^{2} /\left\|\mathbf{e}_{\theta_{1}}\right\|^{2}\left\|\mathbf{e}_{1}\right\|^{2}\right)$ and $\operatorname{dist}\left(\mathcal{E}_{v_{1}}, \mathcal{E}\right)=$ $\sin ^{2}(\Theta)=1-\left(\left|\mathbf{e}_{v_{1}}^{H} \mathbf{e}_{1}\right|^{2} /\left\|\mathbf{e}_{v_{1}}\right\|^{2}\left\|\mathbf{e}_{1}\right\|^{2}\right)$ with $\Omega$ and $\Theta$, respectively, being the largest canonical angles in $[0, \pi / 2]$ between onedimensional linear subspaces $\mathcal{E}_{\theta_{1}}=\left\{\mathbf{e}_{\theta_{1}}\right\}, \mathcal{E}_{v_{1}}=\left\{\mathbf{e}_{v_{1}}\right\}$, and the linear subspace $\mathcal{E}=\left\{\mathbf{e}_{1}\right\}$.

\subsection{Special Case of a Nonmoving Target with Interference}

For a detailed analysis of the TR CRB expression, this section assumes a simplified model consisting of one non-moving target and one unknown deterministic interference source as follows

$$
\mathbf{z}={\tilde{\alpha_{1}}}^{\mathrm{TR}} \mathbf{g}_{1}^{\mathrm{TR}}+{\tilde{\alpha_{3}}}^{\mathrm{TR}} \mathbf{g}_{3}^{\mathrm{TR}}+\mathbf{u}
$$

Since the second target is denoted by index 2 , we represent the interference as the third source. In order to get a closed form expression of the CRB, we assume that $\tilde{\alpha}_{1}^{\mathrm{TR}}$ and $\tilde{\alpha}_{3}^{\mathrm{TR}}$ are known. Directions $\theta_{1}$ and $\theta_{3}$ are unknown. The TR CRB of the target's direction $\theta_{1}$ is

$$
\begin{gathered}
\operatorname{CRB}_{2}^{\mathrm{TR}}\left(\theta_{1}\right)=\left(j_{\theta_{1} \theta_{1}}^{\mathrm{TR}}-\frac{\left|\mathrm{J}_{\theta_{1} \theta_{3}}^{\mathrm{TR}}\right|^{2}}{j_{\theta_{3} \theta_{3}}^{\mathrm{TR}}}\right)^{-1}, \text { with } \\
j_{\theta_{i} \theta_{i}}^{\mathrm{TR}}=4 \mathcal{C}_{\mathrm{TR}}\left(P \frac{4 \pi^{2} \cos ^{2}\left(\theta_{i}\right) \tilde{\kappa}_{T}}{\lambda^{2}}+\frac{4 \pi^{2} \cos ^{2}\left(\theta_{i}\right) \kappa_{T}}{\lambda^{2}}\right) i=1,3, \\
j_{\theta_{1} \theta_{3}}^{\mathrm{TR}}=\frac{2{\tilde{\alpha_{1}}}^{\mathrm{TR} *}{\tilde{\alpha_{3}}}^{\mathrm{TR}} \Re\left\{\frac{\partial \mathbf{g}_{1}^{\mathrm{TR}}{ }^{H}}{\partial \theta_{1}} \frac{\partial \mathbf{g}_{3}^{\mathrm{TR}}}{\partial \theta_{3}}\right\},}{\sigma_{u}^{2}},
\end{gathered}
$$

with $\kappa_{T}=\sum_{i=1}^{P} d_{i}^{T}$ and $\tilde{\kappa}_{T}=\sum_{i=1}^{P}\left(d_{i}^{T}\right)^{2}$. If the target and interference directions $\left\{\zeta_{1}, \zeta_{3}\right\}$ are decoupled, the closed form CRB of $\zeta_{1}$ is

$$
\mathrm{CRB}_{2}^{\mathrm{TR}}\left(\zeta_{1}\right)=\frac{1}{4 \mathcal{C}_{\mathrm{TR}} P^{2} \pi^{2} \sigma_{T}^{2}} \text { with } \sigma_{T}^{2}=\frac{4}{P \lambda^{2}}\left(\tilde{\kappa}_{T}-\frac{\kappa_{T}^{2}}{P}\right)(13)
$$

being the sample variance of the sensor positions in the transmit array and is equal to $4 \tilde{\kappa}_{T} /\left(P \lambda^{2}\right)$ for centro-symmetric arrays.

Result 1. At equal SINR, the CRB of $\theta_{1}$ based on the TR observations (Eq. (12)) is less than the CRB of $\theta_{1}$ based on the conventional $M I M O$ radar in the presence of one interference source.

Proof of Result 1. The CRB of $\theta_{1}$ based on conventional MIMO, takes exactly the form of Eq. (12) except for a difference in the coefficient factors. Then, the TR gain defined as the ratio of the conventional $\operatorname{CRB}\left(\theta_{1}\right)$ to the $\operatorname{CRB}^{\mathrm{TR}}\left(\theta_{1}\right)$ is given as

$\mathcal{G}_{1 I}^{\mathrm{TR}}\left(\theta_{1}\right) \triangleq \frac{\mathrm{CRB}_{2}\left(\theta_{1}\right)}{\mathrm{CRB}_{2}^{\mathrm{TR}}\left(\theta_{1}\right)}=\frac{\mathcal{C}_{\mathrm{TR}}}{\mathcal{C}}=1+\frac{P^{2} c^{2}\left|\tilde{\alpha}_{3}\right|^{2}\left|\left(\left|\tilde{\alpha}_{3}\right|^{2}-\left|\tilde{\alpha}_{1}\right|^{2}\right)\right|}{\sigma_{u}^{2}}$.

Eq. (14) is based on comparing the ratio of $\frac{\mathcal{C}_{\mathrm{TR}}}{\mathcal{C}}$ under same SINR for both the conventional and TR observations.

The second factor in Eq. (14) is directly proportional to the TR normalization factor $c$ and the number of antenna elements $P$ of the array while holding an inverse relationship with the strength (variance) of the observation noise. What is however more interesting to observe is the dependence of the TR gain in the second factor on the attenuations associated with the target and the interference source. Term $\left|\tilde{\alpha}_{3}\right|^{2} \mid\left(\left|\tilde{\alpha}_{3}\right|^{2}-\left|\tilde{\alpha}_{1}\right|^{2} \mid\right.$ maximizes when $\left|\tilde{\alpha}_{3}\right|^{2}=0.5\left|\tilde{\alpha}_{1}\right|^{2}$ with a value approaching 0 when $\left|\tilde{\alpha}_{3}\right|=0$ and $\left|\tilde{\alpha}_{1}\right|=\left|\tilde{\alpha}_{3}\right|$. It is obvious that the multipath factor should disappear in such a case. When $\left|\tilde{\alpha}_{1}\right|=\left|\tilde{\alpha}_{3}\right|$, both the target and the interference have equal attenuation factors. In such a case, it is not possible to differentiate the target and interference responses.

\section{ARL FOR THE TR MIMO RADAR}

In this section, we derive and analyze the TR ARL for both clutterfree and a single interference source models. In terms of the earlier notation, the ARL is defined as $\delta=\zeta_{2}-\zeta_{1}$.

\subsection{TR ARL with No Interference}

With no interference, Eq. (7) can be represented as

$$
\mathbf{z}=\overbrace{{\tilde{\alpha_{1}}}^{\mathrm{TR}} \mathbf{g}_{1}^{\mathrm{TR}}+{\tilde{\alpha_{2}}}^{\mathrm{TR}} \mathbf{g}_{2}^{\mathrm{TR}}}^{\mathbf{z}_{t}}+\mathbf{u} .
$$

Then, the TR ARL is the solution to the Smith's equation [21], i.e., $\delta=\eta \sqrt{\mathrm{CRB}_{2 t}^{\mathrm{TR}}(\delta)}$ (the probabilities of detection and false alarm are selected such that $\eta=1$ ), where $\mathrm{CRB}_{2 t}^{T R}(\delta)$ is the TR CRB associated with model (15). The closed from solution of the ARL involves the analytic inversion of the FIM which is difficult to derive. Instead, we use a partially known radar model [28] to derive the $\mathrm{CRB}$. In this model, it is assumed that both velocity $v_{2}$ and direction $\zeta_{2}$ of the second target are known. Then, the vector of unknown parameter $\tilde{\boldsymbol{\Phi}}_{t}=\left[\begin{array}{llll}\zeta_{1} & v_{1} & \alpha_{1}^{\mathrm{TR}} & \alpha_{2}^{\mathrm{TR}}\end{array}\right]^{T}$. Consequently, the CRB of $\tilde{\boldsymbol{\Phi}}_{t}$ is

$\mathrm{CRB}_{2 t}^{\mathrm{TR}}\left(\zeta_{1}\right)=\tilde{\mathbf{J}}^{\mathrm{TR}}{ }^{-1}$ where $\tilde{\mathbf{J}}^{\mathrm{TR}}{ }^{-1}=\frac{2}{\sigma_{u}^{2}} \mathcal{R}\left\{\left(\frac{\partial \mathbf{z}_{t}}{\partial \tilde{\boldsymbol{\Phi}}_{t}}\right)^{H}\left(\frac{\partial \mathbf{z}_{t}}{\partial \tilde{\boldsymbol{\Phi}}_{t}}\right)\right\}$.

As $\zeta_{2}$ is known and the CRB is the lower bound of an unbiased estimator, then $\mathrm{CRB}_{2 t}^{\mathrm{TR}}(\delta)=\mathrm{CRB}_{2 t}^{\mathrm{TR}}\left(\zeta_{1}\right)$. Since the TR observations (Eq. (15)) follow the 3-way noisy PARAFAC model [24], we use the results presented in Eq. (45) of [19] to derive $\mathrm{CRB}_{2 t}^{\mathrm{TR}}\left(\zeta_{1}\right)$ as

$$
\mathrm{CRB}_{2 t}^{\mathrm{TR}}\left(\zeta_{1}\right) \triangleq \mathrm{CRB}_{1}^{\mathrm{TR}}\left(\zeta_{1}\right)+\frac{\sigma_{u}^{2}}{2 P^{2} K} h^{\mathrm{TR}}(\delta),
$$

where $\mathrm{CRB}_{1}^{\mathrm{TR}}\left(\zeta_{1}\right)$ is the lower bound when there is one target and no interference and $h^{\mathrm{TR}}(\delta)$ takes care of all the elements of the CRB which are a function of the ARL. For a nonmoving radar, centrosymmetric arrays, and high $\mathrm{SNR}, h^{\mathrm{TR}}(\delta)$ is given as [19]

$$
\begin{aligned}
h^{\mathrm{TR}}(\delta) & =\frac{4\left|\tilde{\alpha}_{1}^{\mathrm{TR}}\right|^{2}}{\sigma_{u}^{4}}\left(\mathrm{CRB}_{1}^{\mathrm{TR}}\left(\zeta_{1}\right)\right)^{2}\left|\mathbf{g}_{\zeta_{1}}^{\mathrm{TR}} \mathbf{g}_{2}^{\mathrm{TR}}\right|^{2}(17) \\
\text { where } \mathbf{g}_{\zeta_{1}}^{\mathrm{TR}^{H}} \mathbf{g}_{2}^{\mathrm{TR}} & =2 p^{\mathrm{TR}}(\delta) \Re\left\{\tilde{p}_{T}^{\mathrm{TR}}(\delta) p_{T}^{\mathrm{TR}}(\delta)\right\}
\end{aligned}
$$

and the following notations are used based on the first-order Taylor series expansion in a neighborhood of $\delta=0$ :

$$
\begin{aligned}
& p_{T}^{\mathrm{TR}}(\delta)=\mathbf{a}_{T}^{T}\left(\zeta_{1}\right) \mathbf{a}_{T}^{*}\left(\zeta_{2}\right) \simeq P-j(2 \pi / \lambda) \kappa_{T} \delta \\
& \tilde{p}_{T}^{\mathrm{TR}}(\delta)=\left(\frac{\partial \mathbf{a}_{T}\left(\zeta_{1}\right)}{\partial \zeta_{1}}\right)^{H} \mathbf{a}_{T}\left(\zeta_{2}\right) \simeq\left(4 \pi^{2} / \lambda^{2}\right) \tilde{\kappa}_{T} \delta-(2 j \pi / \lambda) \kappa_{T} \\
& p^{\mathrm{TR}}(\delta)=\mathbf{a}^{\mathrm{TR}}\left(v_{1}\right) \mathbf{a}^{\mathrm{TR}}\left(v_{2}\right)=\sum_{k=0}^{K-1} c_{k}^{2} e^{j 8 \pi T_{p} / \lambda\left(v_{2}-v_{1}\right) k} .
\end{aligned}
$$

Assuming centro-symmetric arrays $\left(\kappa_{T}=0\right)$ and after simplifying, the magnitude square of the modulus term in (17) is expressed as

$$
\left|\mathbf{g}_{\zeta_{1}}^{\mathrm{TR}^{H}} \mathbf{g}_{2}^{\mathrm{TR}}\right|^{2}=4 c^{2} P^{4} \pi^{4}\left|s_{\mathrm{TR}}\right|^{2} \sigma_{T}^{4} \delta^{2}
$$

where $p^{\mathrm{TR}}(\delta)=c^{2} s_{\mathrm{TR}}$. Using the Smith's criterion $\delta=\sqrt{\mathrm{CRB}_{2 t}^{\mathrm{TR}}\left(\zeta_{1}\right)}$ and using Eq. (16), the TR ARL expression is given as

$$
\delta=\sqrt{\frac{\mathrm{CRB}_{1}^{\mathrm{TR}}\left(\zeta_{1}\right)}{1-\left[\left(8 P^{2} \pi^{4}\left|s_{\mathrm{TR}}\right|^{2} \sigma_{T}^{4} / K\right)\left(\mathcal{C}_{\mathrm{TR}}\right)\left(\mathrm{CRB}_{1}^{\mathrm{TR}}\left(\zeta_{1}\right)\right)^{2}\right]}} .
$$

Substituting the lower bound in the absence of clutter in Eq. (21) and assuming high SNR, the TR ARL will be simplified to $\sqrt{\mathrm{CRB}_{1}^{\mathrm{TR}}\left(\zeta_{1}\right)}$. Consequently, the TR ARL has the same properties as TR CRB. 


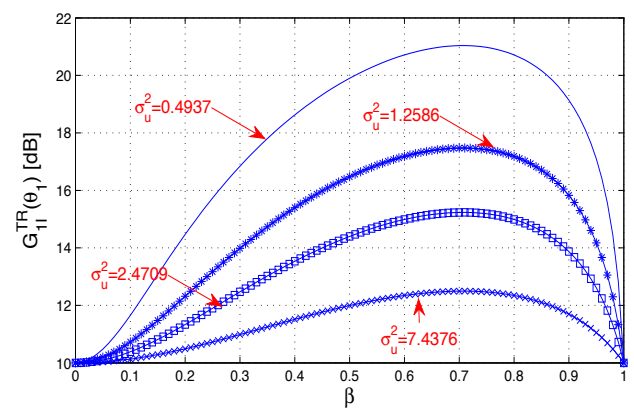

Fig. 1. TR CRB gain as function of interference and noise.

\subsection{TR ARL of Nonmoving Targets with Interference}

Assume a model based on two targets and one interference source. The unknown parameters are $\zeta_{1}, \zeta_{3}$ and $\tilde{\alpha_{2}}$. Rest of the parameters are assumed known. The FIM for the unknown parameters is

$$
\mathbf{J}^{\mathrm{TR}}=\frac{2}{\sigma_{u}^{2}}\left[\begin{array}{cc}
\mathbf{J}_{\zeta}^{\mathrm{TR}} & \mathbf{B}(\delta) \\
\mathbf{B}^{T}(\delta) & P^{2},
\end{array}\right]
$$

where $\mathbf{J}_{\zeta}^{\mathrm{TR}}$ is the FIM derived in Section 3.2 for one target and one interference; unknown parameters $\zeta_{1}=\sin \left(\theta_{1}\right)$ and $\zeta_{3}=\sin \left(\theta_{3}\right)$; and

$$
\mathbf{B}(\delta)=\left[\begin{array}{c}
j_{\zeta_{1}}^{\mathrm{TR}} \tilde{\alpha}_{2} \\
j_{\zeta_{3} \tilde{\alpha_{2}}}^{\mathrm{TR}}
\end{array}\right]=\left[\begin{array}{l}
\Re\left\{\tilde{\alpha_{1}}{ }^{*}\left(\partial \mathbf{g}_{1} / \partial \zeta_{1}\right)^{H} \mathbf{g}_{2}\right\} \\
\Re\left\{\tilde{\alpha_{3}}{ }^{*}\left(\partial \mathbf{g}_{3} / \partial \zeta_{3}\right)^{H} \mathbf{g}_{2}\right\}
\end{array}\right] .
$$

Using the Schur complement inverse of $\mathbf{J}^{\mathrm{TR}}$, the TR CRB of $\zeta_{1}$ is

$$
\begin{aligned}
\mathrm{CRB}_{3}^{\mathrm{TR}}\left(\zeta_{1}\right) & =\mathrm{CRB}_{2}^{\mathrm{TR}}\left(\zeta_{1}\right)+\frac{\sigma_{u}^{2}}{2 P^{2}} h_{2}^{\mathrm{TR}}(\delta), \\
\text { where } \quad h_{2}^{\mathrm{TR}}(\delta) & =\left[\mathbf{J}_{\zeta}^{\mathrm{TR}}{ }^{-1} \mathbf{B}(\delta) \mathbf{B}^{T}(\delta) \mathbf{J}_{\zeta}^{\mathrm{TR}}\right]_{11}^{-1}
\end{aligned}
$$

In Eq. (24), the TR CRB of $\zeta_{1}$ based on one target and one interference is denoted by $\mathrm{CRB}_{2}^{\mathrm{TR}}\left(\zeta_{1}\right)$ and that of the two targets and one interference source is denoted by $\operatorname{CRB}_{3}^{\mathrm{TR}}\left(\zeta_{1}\right)$. Defining notations $\mathcal{C}_{1}^{\mathrm{TR}}=c^{2}\left|\tilde{\alpha}_{1}^{\mathrm{TR}}\right|^{2} / \sigma_{u}^{2}$ and $\mathcal{C}_{3}^{\mathrm{TR}}=c^{2}\left|\tilde{\alpha}_{3}^{\mathrm{TR}}\right|^{2} / \sigma_{u}^{2}$, Eq. (25) simplifies to

$h_{2}^{\mathrm{TR}}(\delta)=$

$\left(\frac{\sqrt{2 \mathcal{C}_{1}^{\mathrm{TR}}}}{\sigma_{u}} \mathrm{CRB}_{2}^{\mathrm{TR}}\left(\zeta_{1}\right)\left|\mathbf{g}_{\zeta_{1}}^{\mathrm{TR}}{ }^{H} \mathbf{g}_{2}^{\mathrm{TR}}\right|+\frac{\sqrt{2 \mathcal{C}_{3}^{\mathrm{TR}}}}{\sigma_{u}} \mathrm{CRB}_{2}^{\mathrm{TR}}\left(\zeta_{1}, \zeta_{3}\right)\left|\mathbf{g}_{\zeta_{3}}^{\mathrm{TR}}{ }^{H} \mathbf{g}_{2}^{\mathrm{TR}}\right|\right)^{2}$.

Note that $\left|\mathbf{g}_{\zeta_{1}}^{\mathrm{TR}} \mathbf{g}_{2}^{\mathrm{TR}}\right|=\left(2 \pi^{2} \sigma_{T}^{2} P^{2}\right) \delta$. Rearrange terms in (24) as

$$
\begin{aligned}
& \left(\mathcal{C}_{1}^{\mathrm{TR}} 4 \pi^{2} \sigma_{T}^{4} P^{2} \mathrm{CRB}_{2}^{\mathrm{TR}}\left(\zeta_{1}\right)-1\right) \delta^{2}+ \\
& \left(4 \sqrt{\mathcal{C}_{1}^{\mathrm{TR}} \mathcal{C}_{3}^{\mathrm{TR}}} \pi^{2} \sigma_{T}^{2} \gamma\right) \delta+\mathrm{CRB}_{2}^{\mathrm{TR}}\left(\zeta_{1}\right)+\frac{\mathcal{C}_{3}^{\mathrm{TR}}}{P^{2}} \mathrm{CRB}_{2}^{\mathrm{TR}}\left(\zeta_{1}, \zeta_{3}\right) \gamma^{2}=0,
\end{aligned}
$$

where $\gamma=\left|\mathbf{g}_{\zeta_{3}}^{\mathrm{TR}} \mathbf{g}_{2}^{\mathrm{TR}}\right|$. The ARL $\delta$ is the solution of the above polynomial. In order to compare the TR ARL with the conventional one, we assume that the target direction $\zeta_{1}$ and the interference direction $\zeta_{3}$ are decoupled. Therefore, the second term in $h_{2}^{\mathrm{TR}}$ reduces to zero.

Result 2. At equal SINR and with one interference, the TR ARL is less than the ARL based on the conventional MIMO radar.

Proof of Result 2. Using Eq. (24) and based on the fact that $h_{2}^{\mathrm{TR}}=$ $\left(2 \mathcal{C}_{1}^{\mathrm{TR}} / \sigma_{u}^{2}\right)\left(4 \pi^{4} \sigma_{T}^{4} P^{4}\right) \mathrm{CRB}_{2}^{\mathrm{TR}}{ }^{2}\left(\zeta_{1}\right) \delta^{2}$, the TR ARL is given by

$$
\delta=\sqrt{\frac{\mathrm{CRB}_{2}^{\mathrm{TR}}\left(\zeta_{1}\right)}{1-\left[\left(4 P^{2} \pi^{4} \sigma_{T}^{4}\right)\left(\mathcal{C}_{\mathrm{TR}}\right)\left(\mathrm{CRB}_{2}^{\mathrm{TR}}\left(\zeta_{1}\right)\right)^{2}\right]}} .
$$

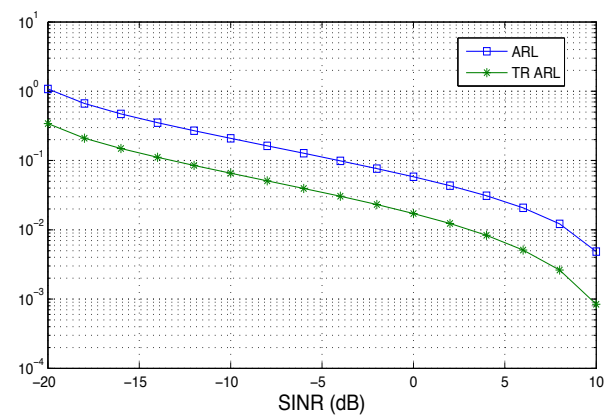

Fig. 2. ARLs for conventional and TR MIMO arrays.

Substituting the expression of $\mathrm{CRB}_{2}^{\mathrm{TR}}\left(\zeta_{1}\right)$ from (13), the TR ARL in presence of one interference source is given as

$$
\delta=\sqrt{\frac{1}{\pi^{2} \sigma_{T}^{2}\left(4 \mathcal{C}_{\mathrm{TR}} P^{2}-1\right)}} .
$$

At high SNR, $\mathcal{C}_{\mathrm{TR}} P^{2} \gg 1$ and the ARL simplifies to $\sqrt{\mathrm{CRB}_{2}^{\mathrm{TR}}\left(\zeta_{1}\right)}$. Based on Result 1 , the ratio of the conventional ARL to the TR ARL is $\sqrt{\mathcal{G}_{1 I}^{\mathrm{TR}}\left(\zeta_{1}\right)}$ which is greater than one. Therefore, the TR ARL is always less than the conventional ARL at equal SINR.

\section{NUMERICAL ILLUSTRATION}

This section demonstrates the potential performance gain of the TR/MIMO framework by numerical examples. One colocated Uniform Linear Arrays (ULA) with 10 antenna elements and halfwavelength inter-element spacing is used as transmit and receive arrays. The targets are located at $\zeta_{1}=\sin (\pi / 6)=0.5$ and at $\zeta_{2}=0.51$ with respect to the broadside of the array. The direction of arrival associated with the interference source is set to $\zeta_{3}=\sin (\pi / 4)$. The attenuation factors for the two targets and one interference source are set to $[0.8,0.8,0.4]^{T}$, respectively. Attenuations are assumed to be real to simplify the matrix inversion operation needed to derive the CRBs. In Fig. 1, we plot the TR gain for the direction of arrival $\theta_{1}$ as given in Result 1 for a fixed direct path attenuation of $\left|\tilde{\alpha_{1}}\right|=0.8$ and different values of $\left|\tilde{\alpha_{3}}\right|=\beta\left|\tilde{\alpha_{1}}\right|$ for $(0<\beta \leq 1)$. The variance of noise in TR stage (i.e. $\left.\sigma_{u}^{2}\right)$ is varied and the TR gain is plotted for different noise variances. As shown in this figure, the gain is maximum for $\beta=\frac{1}{\sqrt{2}}$ or $\left|\tilde{\alpha_{1}}\right|^{2}=2\left|\tilde{\alpha_{3}}\right|^{2}$, which is in agreement with Result 1. Fig. 2 plots the ARL obtained for the conventional and TR/MIMO systems at the same SINRs set for $\beta=0.5$. As shown in this figure, the TR/MIMO offers lower ARL in a one interference source model. For a fair comparison, we keep the same SINRs for both the conventional and TR frameworks.

\section{SUMMARY}

The paper derives and analyzes closed form expressions for the TR/MIMO CRBs of the direction of arrival associated with a target embedded in a multipath environment corrupted with noise. Our derivations in a 2-path environment reveals that using TR, the CRBs for direction can be improved over the conventional MIMO systems. Finally, the TR ARL is derived for both environments with and without clutter. At high SNR and equal SINR, the TR ARL is shown to be lower than that for the convectional ARL. 


\section{REFERENCES}

[1] P. Blomgren, G. Papanicolaou, and H. Zhao, "Super-resolution in time-reversal acoustics," Journal of the Acoustical Society of America, 111, 230-48, 2001.

[2] C. Tsogka and G. C. Papanicolaou, "Time reversal through a solid-liquid interface and super-resolution," Inverse problems, vol. 18, no. 6, pp. 1639-1657, 2002.

[3] M. Fink, "Time reversal of ultrasonic fields- Part I: Basic principles," IEEE Transactions on Ultrasonics, Ferroelectrics and Frequency Control, vol. 39, no. 5, pp. 555-566, Sep 1992.

[4] Y. Jin and J. Moura, "Time-reversal detection using antenna arrays," IEEE Transactions on Signal Processing, vol. 57, no. 4, pp. $1396-1414$, april 2009.

[5] Y. Jin, J. Moura, and N. O'Donoughue, "Time reversal in multiple-input multiple-output radar," IEEE J. of Sel. Topics in Signal Processing, vol. 4, no. 1, pp. 210 -225, Feb. 2010.

[6] F. Foroozan and A. Asif, "Time reversal based active array source localization," IEEE Trans. on Signal Processing, vol. 59, no. 6, pp. 2655 -2668, June 2011.

[7] — - "Cramér-Rao bound for time reversal active array direction of arrival estimators in multipath environments," Proceedings of the International Conference on Acoustics, Speech, and Signal Processing (ICASSP), pp. 2646-2649, Mar. 2010.

[8] F. Foroozan, A. Asif, Y. Jin, and J. Moura, "Direction finding algorithms for time reversal MIMO radars," IEEE SSP, pp. 433437, 2011.

[9] F. Foroozan and A. Asif, "Time reversal MIMO radars for estimation of velocity and direction," IEEE SSP, accepted, 2012.

[10] _ _ "Information theoretic bounds for angle-doppler estimation in time reversal MIMO communication," IEEE Globecom, accepted, 2012.

[11] J. Tabrikian, Performance Bounds and Techniques for Target Localization Using MIMO Radars, Chapter 4 in MIMO Radar Signal Processing, J. Li and P. Stoica, Eds. Wiley, 2009.

[12] E. Fishler, A. Haimovich, R. Blum, J. Cimini, L.J., D. Chizhik, and R. Valenzuela, "Spatial diversity in radars-models and detection performance," IEEE Transactions on Signal Processing, vol. 54, no. 3, pp. 823 - 838, mar. 2006.

[13] V. Mecca, D. Ramakrishnan, and J. Krolik, "MIMO radar space-time adaptive processing for multipath clutter mitigation," Fourth IEEE Workshop on Sensor Array and Multichannel Processing, pp. 249 -253, Jul. 2006.

[14] L. Xu, J. Li, and P. Stoica, "Adaptive techniques for MIMO radar," Fourth IEEE Workshop on Sensor Array and Multichannel Processing, pp. 258 -262, jul. 2006.

[15] L. Xu and J. Li, "Iterative generalized-likelihood ratio test for MIMO radar," IEEE Transactions on Signal Processing, vol. 55, no. 6, pp. 2375 -2385, June 2007.

[16] S. M. Kay, Fundamentals of statistical signal processing: estimation theory. Upper Saddle River, NJ, USA: Prentice-Hall, Inc., 1993.

[17] I. Bekkerman and J. Tabrikian, "Target detection and localization using MIMO radars and sonars," IEEE Transactions on Signal Processing, vol. 54, no. 10, pp. 3873 -3883, October 2006.
[18] A. Dogandzic and A. Nehorai, "Cramer-Rao bounds for estimating range, velocity, and direction with an active array," IEEE Transactions on Signal Processing, vol. 49, no. 6, pp. 1122 -1137, Jun. 2001.

[19] R. Boyer, "Performance bounds and angular resolution limit for the moving colocated MIMO radar," IEEE Transactions on Signal Processing, vol. 59, no. 4, pp. 1539 -1552, april 2011.

[20] A. Swindlehurst and P. Stoica, "Maximum likelihood methods in radar array signal processing," Proceedings of the IEEE, vol. 86, no. 2, pp. $421-441,1998$.

[21] S. Smith, "Statistical resolution limits and the complexified Cramér-Rao bound," IEEE Transactions on Signal Processing,, vol. 53, no. 5, pp. 1597 - 1609, May 2005.

[22] H. Godrich, A. M. Haimovich, and R. S. Blum, "Target localization accuracy gain in MIMO radar based systems," IEEE Trans. on Inf. Theory, vol. 56, no. 6, pp. 2783 -2803, 2010.

[23] A. J. Dekker and A. Bos, "Resolution, a survey," J. Opt. Soc. Am., vol. A, no. 14, pp. 547 -557, 1997.

[24] D. Nion and N. Sidiropoulos, "A PARAFAC-based technique for detection and localization of multiple targets in a MIMO radar system," in ICASSP 2009., april 2009, pp. 2077 -2080.

[25] C. Chen and P. Vaidyanathan, "Mimo radar space-time adaptive processing using prolate spheroidal wave functions," IEEE Transactions on Signal Processing, vol. 56, no. 2, pp. 623-635, 2008.

[26] Y. Jin, J. Moura, N. O'Donoughue, and J. Harley, "Single antenna time reversal detection of moving target," in IEEE ICASSP, March 2010, pp. 3558 -3561.

[27] P. Stocia and R. Moses, Introduction to Spectral Analysis. Prentice Hall, New Jersey, 1997.

[28] Z. Liu and A. Nehorai, "Statistical angular resolution limit for point sources," IEEE Transactions on Signal Processing, vol. 55, no. 11, pp. $5521-5527$, nov. 2007. 